\title{
Effect of endophytic Bacillus subtilis on drought stress tolerance of Triticum aestivum L. plants of Steppe Volga and Forest-Steppe West Siberian agroecological groups Lastochkina O. ${ }^{1,2}$, Garshina D. ${ }^{2}$, Pusenkova L. ${ }^{2}$
}

${ }^{1}$ Institute of Biochemistry and Genetics - Subdivision of the Ufa Federal Research Center of the Russian Academy of Sciences, Ufa, Russia; ${ }^{2}$ Bashkir Research Institute of Agriculture - Subdivision of the Ufa Federal Research Center of the Russian Academy of Sciences, Ufa, Russia

E-mail: oksanaibg@gmail.com

Key message. Physiological responses of wheat to B. subtilis under drought depends on the belonging of plants to agroecological groups. B. subtilis showed the best positive effect on growth and water status of Steppe Volga agroecological group's wheat.

Keywords: endophytic Bacillus subtilis, wheat, agroecological groups, tolerance, drought

Drought is one of the major abiotic stresses worldwide leading to inhibition of growth and reduction the crop yield, including wheat. Endophytic bacteria Bacillus subtilis are considering as promising bio-safe and eco-friendly strategies to cope with adverse abiotic stresses in plants. However, underlying mechanisms of growth-stimulating and anti-stress physiological programs of $B$. subtilis still far from clear. The aim of this study was to investigate the effect of inoculation with $B$. subtilis (strain 10-4 and 26D) on growth (seed germination, length of roots and shoots) and water-holding capacity (WHC) of wheat plants belonging to different agroecological groups (Steppe Volga (SV) and Forest Steppe West Siberia (FSWS), genetically having different drought stress adaptation strategies) under drought stress conditions. The experiments were carried out on wheat (Triticum aestivum L.) plants (cultivars Saratovskaya-55, Ekada-70 - representatives of SV agroecological group and Omskaya-35, Salavat Yulaev - representatives of FSWS agroecological group). The seeds before sowing was inoculated with B. subtilis 10-4 $\left(10^{5} \mathrm{CFU} / \mathrm{ml}\right)$ and $26 \mathrm{D}\left(10^{8} \mathrm{CFU} / \mathrm{ml}\right)$ (Lastochkina et al. 2017). Growth parameters and WHC of leaves were determined according to (Mokronosov 1994; Udovenko 1988). Drought stress was modulated by 12\% PEG-6000. It was revealed that pre-sowing inoculation with B. subtilis 10-4 and 26D exerted a multidirectional character of the effects on the drought tolerance of wheat plants of the studied agroecological groups at the initial stages of ontogenesis. B. subtilis (10-4, 26D) contributed to better seed germination, and increased length of roots and shoots of wheat seedlings of the $C v$. Saratovskaya-55 and Ekada-70 while practically did not, or, conversely, in some cases, even inhibited the same growth indices for wheat $C v$. Omskaya-35 and Salavat Yulaev under drought stress. It was found that the ability of B. subtilis (10-4, 26D) to influence on the growth indices of wheat cultivars of different ecotypes has a correlation with Bacillus-induced changes in their leaf's WHC. The impact of drought reduced WHC of $C v$. Omskaya-35 and Salavat Yulaev plants by 2-2.5 times, while for the $C v$. Saratovskaya-55 and Ekada-70, the indices of WHC decreased slightly. Inoculation with B. subtilis (10-4, 26D) increased (under normal condition) the WHC of leaf tissues of all studied plants, and the $C v$. Saratovskaya-55 and Ekada-70 showed the greatest responsiveness to strains 10-4 and 26D inoculation, both under normal and drought stress conditions. It was revealed that $B$. subtilis $10-4,26 \mathrm{D}$ prevented drought-induced decrease in WHC of leaves for $C v$. Saratovskaya-55 and Ekada-70, and reduced for $C v$. Omskaya-55 and Salavat Yulaev. Thus, the findings indicate a correlation between the growth parameters and leaf' WHC as well as participation of $B$. subtilis in maintaining water status in plants under droughts stress and initiation of protective reactions, especially for $C v$. Ekada-70 and Saratovskaya-55, which showed the greatest positive responsiveness in response to $B$. subtilis $(10-4,26 \mathrm{D})$ inoculation under drought stress.

The study was funded by RFBR according to the research project № 19-016-00035.

Lastochkina O., Pusenkova L., Yuldashev R., Babaev M., Garipova S., Blagova D., Khairullin R., Aliniaeifard S. 2017. Effects of Bacillus subtilis on some physiological and biochemical parameters of Triticum aestivum L. (wheat) under salinity. Plant physiology and biochemistry. 121:80-88. doi: 10.1016/j.plaphy.2017.10.020.

Mokronosov A.T. Small workshop on plant physiology/ Ed. MokronosovA.T. Moscow: MSU. 1994. 184 p.

Udovenko G.V. Diagnosis of plant resistance to stresses: methodological guide/ Ed. Udovenko G.V. Leningrad: VIR. 1988. $227 \mathrm{p}$. 